LA-UR-99-3581

Approved for public release;

distribution is unlimited.

\title{
ANALYSIS OF DATA FROM Z-PINCH MTF TARGET PLASMA EXPERIMENTS
}

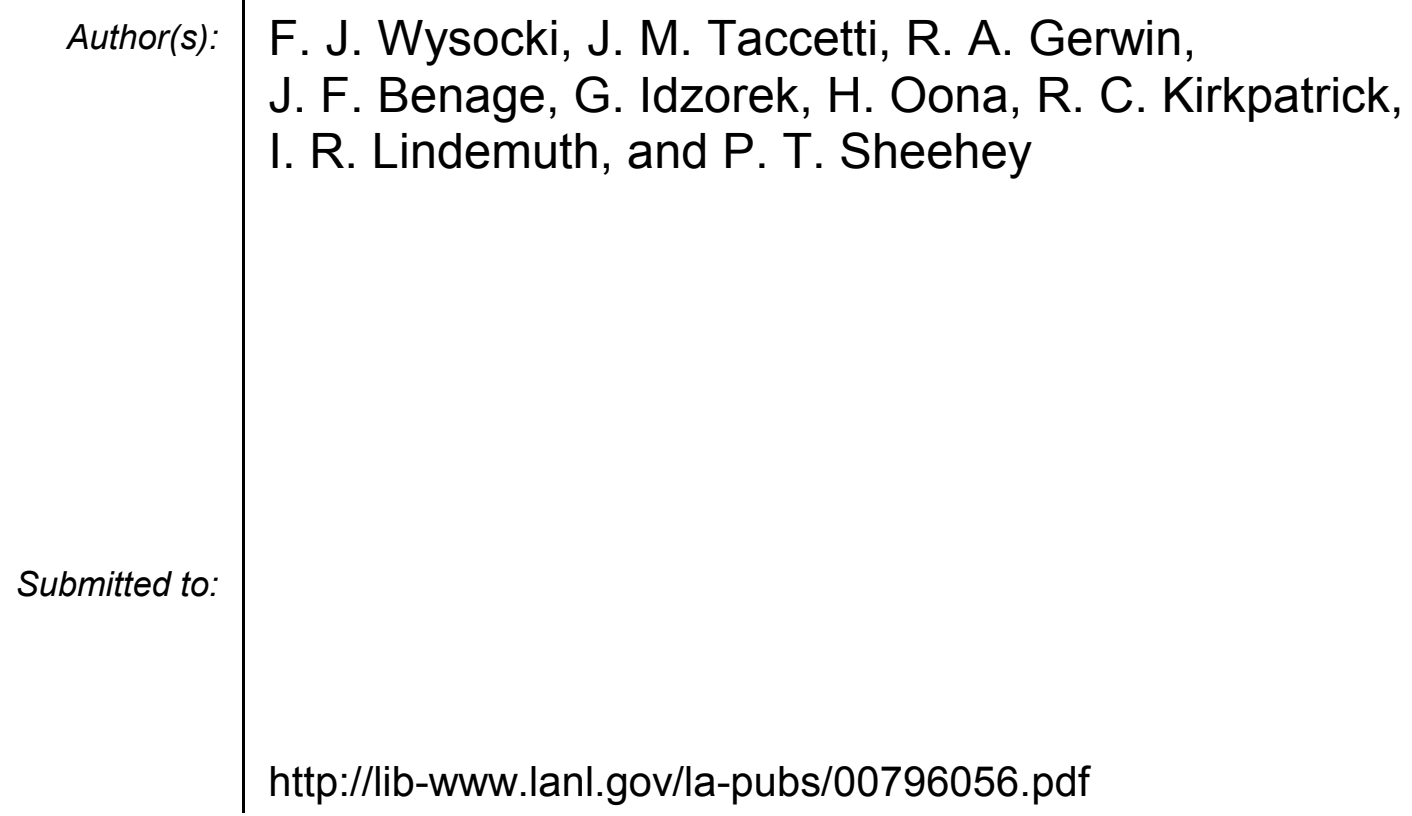

Los Alamos National Laboratory, an affirmative action/equal opportunity employer, is operated by the University of California for the U.S. Department of Energy under contract W-7405-ENG-36. By acceptance of this article, the publisher recognizes that the U.S. Government retains a nonexclusive, royaltyfree license to publish or reproduce the published form of this contribution, or to allow others to do so, for U.S. Government purposes. Los Alamos National Laboratory requests that the publisher identify this article as work performed under the auspices of the U.S. Department of Energy. Los Alamos National Laboratory strongly supports academic freedom and a researcher's right to publish; as an institution, however, the Laboratory does not endorse the viewpoint of a publication or guarantee its technical correctness. 


\title{
ANALYSIS OF DATA FROM Z-PINCH MTF TARGET PLASMA EXPERIMENTS*
}

\author{
F. J. Wysocki, J. M. Taccetti, R. A. Gerwin, J. F. Benage, G. Idzorek, H. Oona, R. C. Kirkpatrick, \\ I. R. Lindemuth, and P. T. Sheehey \\ Los Alamos National Laboratory, PO Box 1663, Los Alamos, New Mexico, 87545, USA
}

\begin{abstract}
The Los Alamos National Laboratory Colt facility has been used to create target plasma for Magnetized Target Fusion (MTF). The primary results regarding magnetic field, plasma density, plasma temperature, and hot plasma lifetime are summarized and the suitability of these plasma targets for MTF is assessed.
\end{abstract}

\section{INTRODUCTION}

Magnetized Target Fusion (MTF) is a two stage approach to achieve fusion conditions [1,2]. First a suitable target plasma is generated in a geometry which allows a subsequent implosion of this plasma (the second stage). The Los Alamos National Laboratory Colt facility has been used to study target plasma created by driving a z-directed current through a plasma contained by a $2 \mathrm{~cm}$ radius by $2 \mathrm{~cm}$ high cylindrical metal wall. The parameters of this plasma system have been studied to evaluate the suitability of this approach for generating target plasma for MTF. The subsequent implosion of this system has not been studied.

The experimental configuration of the electrodes is shown in Fig. 1 of a companion paper in this proceedings [3], and the parameters of the Colt capacitor bank are summarized in Ref. 3. Two modes of operation have been studied; a static gas fill operation described in more detail in Ref. 4, and operation with a polyethylene fiber along the central axis described in more detail in Ref. 5. In this report, the primary results regarding magnetic field, plasma density, and plasma temperature are summarized. In addition, the lifetime of the hot plasma is discussed and scaling of this lifetime with other parameters is presented.

\section{RESULTS}

The primary parameters that a MTF target plasma should have are [1]: (a) an embedded magnetic field, (b) closed magnetic field lines, (c) magnetic field strength of at least $5 \mathrm{~T}$, (d) plasma density of $\approx 10^{18} \mathrm{~cm}^{-3}$, (e) a minimum plasma temperature of $50 \mathrm{eV}$, but $200-300 \mathrm{eV}$ is a more realistic requirement, (f) low impurity content, and (g) configuration lifetime longer than the subsequent implosion time. Data is presented which supports achievement of parameters (a) through (e). The level of impurities is unknown, and data presented indicates the hot plasma lifetime is not long enough for subsequent implosion.

\section{A. Magnetic Field}

The requirement of an embedded magnetic field with closed lines is (nominally) automatically satisfied with the diffuse z-pinch approach. There may be an issue regarding how well the field lines remain closed due to possible magnetic turbulence, but that subject is beyond the scope of this report. Figure 1 shows the achievement of the required $5 \mathrm{~T}$ in these discharges, as measured by $\mathrm{B}$ dot probes inserted along the bottom wall of the plasma containment region (see Fig. 1 in Ref. 3). The radial profile of the magnetic field is consistent with a diffuse zdirected current.

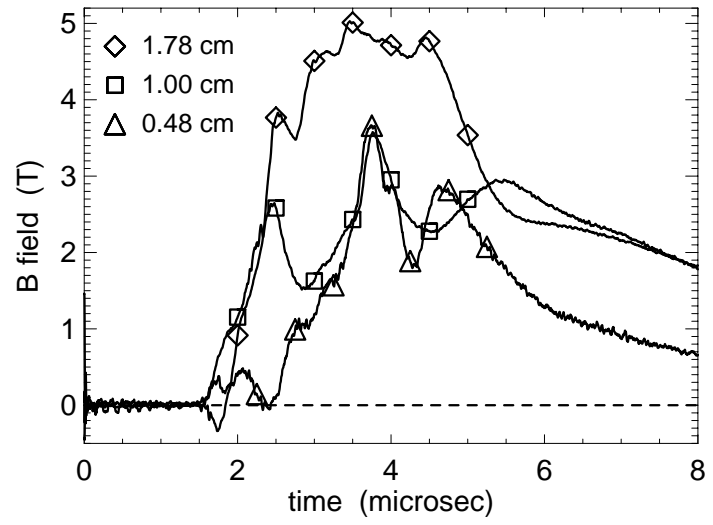

Figure 1. Magnetic field strength measured at three radii at the bottom wall of the plasma containment region.

\section{B. Plasma Density}

A HeCd laser (442 $\mathrm{nm}$ wavelength) interferometer was used to measure the plasma density. The line averaged density along a diameter of the mid-plane of the pinch is shown in Fig. 2. The density is observed to rise steadily in time to roughly the required $10^{18} \mathrm{~cm}^{-3}$. At some point in each discharge, the quadrature interference signal is lost, and subsequent data can not be analyzed. Each curve is truncated at that time point.

\footnotetext{
* Work supported by the US DOE.
} 


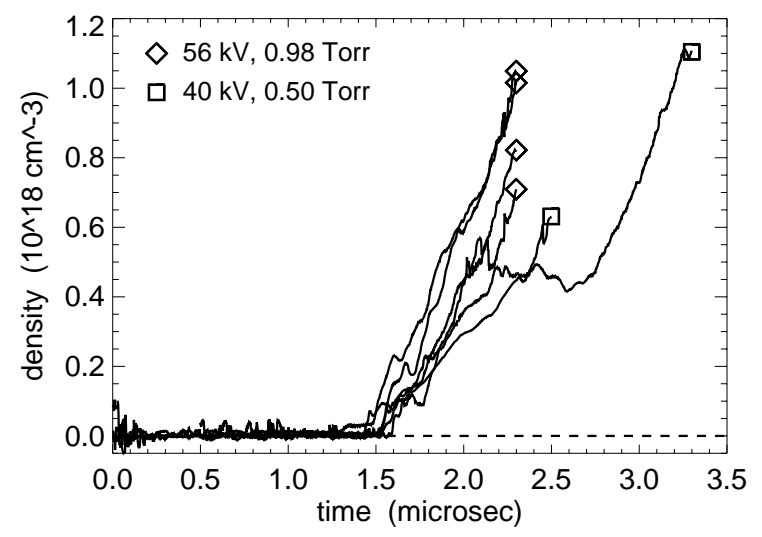

Figure 2. Line averaged plasma density measured with a $\mathrm{HeCd}$ laser interferometer for four discharges at $56 \mathrm{kV}$ charge voltage and 0.98 torr hydrogen static fill, and for two discharges at $40 \mathrm{kV}$ charge voltage and 0.5 torr hydrogen static fill.

\section{Temperature and Hot Plasma Lifetime}

Filtered silicon photodiodes are used to assess the plasma temperature. Formal analysis of that data is presented in a companion paper (Ref. 3). Figures 2 and 3 in Ref. 3 show that the peak plasma temperature easily exceeds the minimum required $50 \mathrm{eV}$, and reaches into the 200-300 eV realistic requirement range. However, as discussed in Ref. 3, this hot plasma does not last more than a few $\mu$ s. To further characterize the hot plasma lifetime properties, a simpler photodiode analysis procedure is used which is independent of any chosen model spectrum. Two diode signals are analyzed, one from a diode without any filter (open diode), and one from a diode with a thick polystyrene filter which only transmits visible light. Typical data from these diodes are shown in Fig. 3. The ratio of these two signals (Fig. 4) is then used as a qualitative measure of the plasma temperature; i.e., when the ratio is large (several hundred), the plasma temperature is high (of order 200

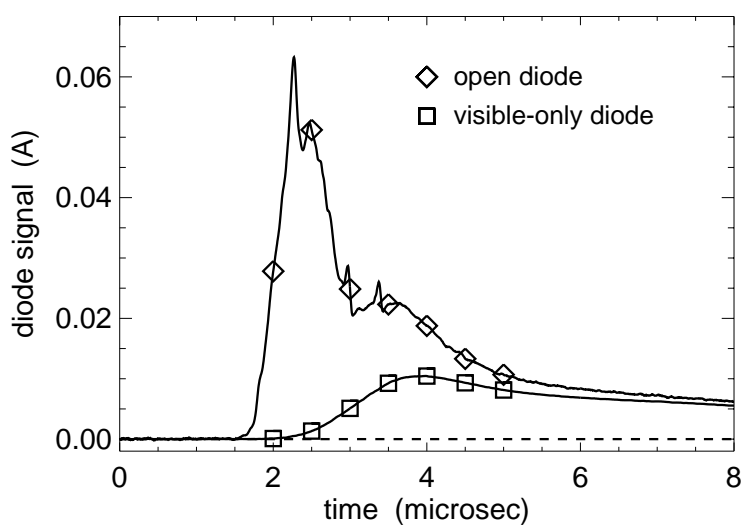

Figure 3. Typical silicon photodiode data for an open diode (no filter) and a diode with a thick polystyrene filter which only transmits visible light.
$\mathrm{eV}$ ) as shown in Ref. 3, and when the ratio tends to one, the plasma has become very cold $(<10 \mathrm{eV})$. A hot plasma lifetime is defined as the time it takes for the open to visible ratio to drop from the maximum value to $5 \%$ of the maximum value.

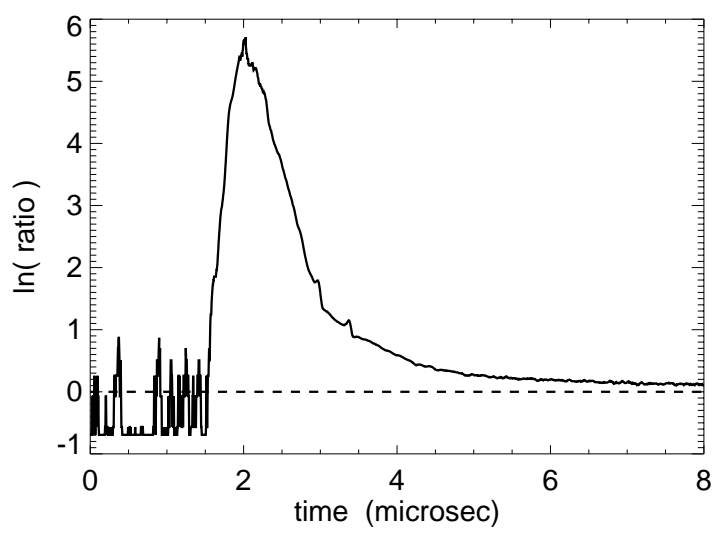

Figure 4. The natural logarithm of the ratio of the open diode signal divided by the visible-only diode signal from the data shown in Fig. 3.

The maximum open diode signal from many discharges as a function of the capacitor bank output voltage is shown in Fig. 5. Data from hydrogen fill, polyethylene fiber, and methane fill discharges are included. For the hydrogen fill discharges, the fill pressure was 0.5 torr for $40 \mathrm{kV}$ bank voltage, and was adjusted to follow the square of the bank voltage at other voltages. The methane fill pressure varied between 0.06 torr to 0.1 torr. Methane fill discharges were performed because methane has a similar carbon contaminate level as polyethylene. Comparing the data from methane fill discharges to data from hydrogen fill discharges gives some indication of the penalty of the carbon contaminate. This then gives some indication of how well a pure hydrogen (or deuterium) fiber discharge would have performed. (Cryogenic deuterium fiber discharges where not performed for technical reasons. [5])

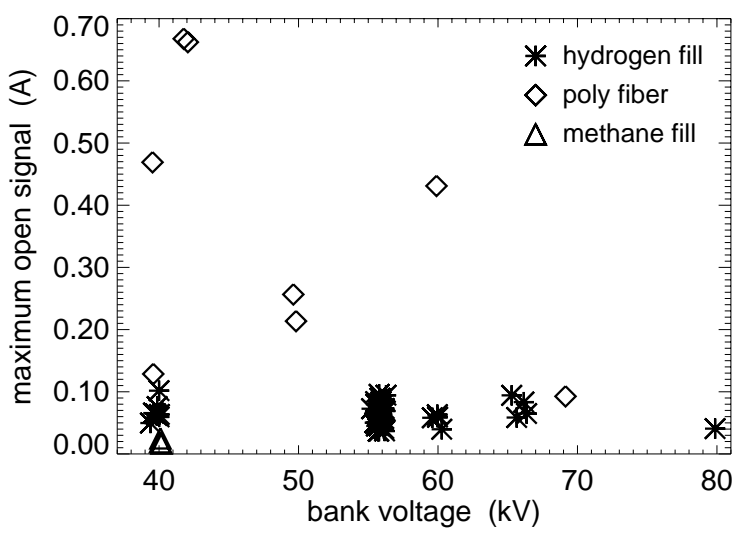

Figure 5. The maximum open diode signal as a function of the capacitor bank output voltage for 54 hydrogen fill discharges, 8 polyethylene fiber discharges, and 3 methane fill discharges. 
The data in Fig. 5 show that the polyethylene fiber discharges produce higher maximum open diode signal compared to the gas fill discharges. This is due to the strong $\mathrm{m}=0$ "sausage" instability events which occur in the fiber discharges but not in the gas fill discharges $[3,4,5]$. The data also shows that, particularly for the hydrogen fill discharges, the maximum open diode signal does not depend on capacitor bank output voltage.

Figure 6 shows the maximum ratio of open diode signal divided by the visible-only diode signal as a function of the capacitor bank output voltage. As stated earlier, the maximum ratio is interpreted as a qualitative measure of the maximum plasma temperature. The data show that while the polyethylene fiber discharges produce a higher maximum open diode signal compared to the gas fill discharges, the maximum ratio, i.e., the plasma temperature, is not higher compared to the gas fill discharges. In addition, the maximum ratio for the methane fill discharges is not substantially lower than for the hydrogen discharges. As before, there is not an

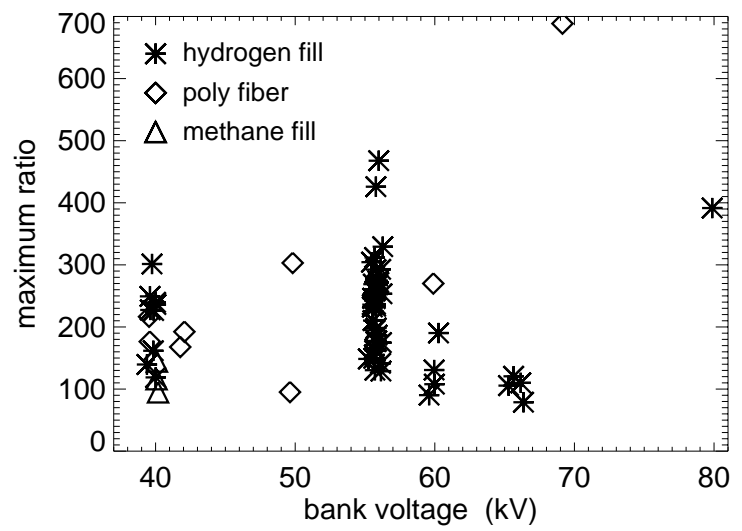

Figure 6. The maximum ratio of the open diode signal divided by the visible-only diode signal as a function of the capacitor bank output voltage for the same discharges shown in Fig. 5.

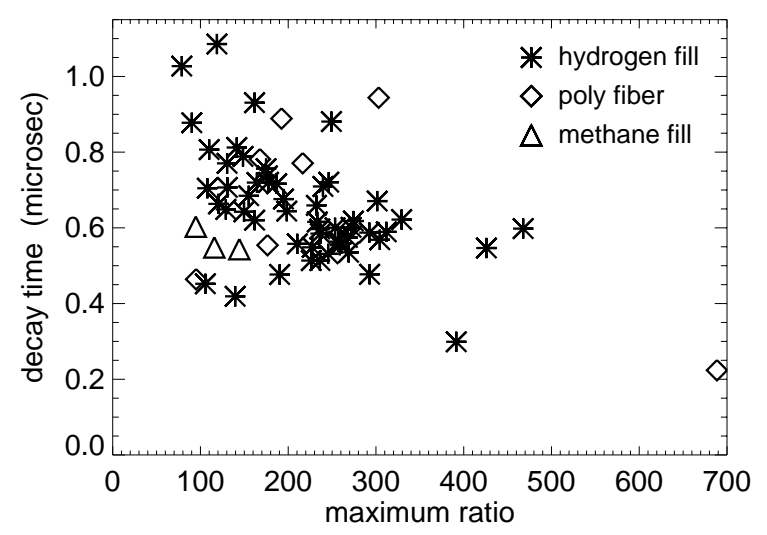

Figure 7. The hot plasma decay time as defined by the time for the ratio of open to visible-only diode signal to drop from the maximum value to $5 \%$ of the maximum value as a function of the maximum ratio for the same discharges as shown in Figs. 5 and 6.

obvious trend with capacitor bank output voltage, but there is a high level of shot-to-shot variation.

A measure of the hot plasma decay time is defined as the time for the ratio to drop from the maximum value to $5 \%$ of the maximum value. These data as a function of the maximum ratio are shown in Fig. 7. The figure shows that the relatively short decay time is universal to all discharges performed. There is large shot-to-shot variation, and the polyethylene and methane discharge data is not significantly different from the hydrogen fill discharge data. Also, it appears that the statistical average of the decay time, particularly for the hydrogen fill discharges, drops somewhat as the maximum ratio increases.

While there is not a good measure of the impurity content in the hydrogen fill discharges, it is interesting to examine the data from a discharge known to be $100 \%$ impure. The open diode and visible-only diode signals from a discharge with $25 \%$ oxygen $/ 75 \%$ argon fill are shown in Fig. 8, plotted with the same $\mathrm{x}$ and $\mathrm{y}$ scales as in Fig. 3. The fill pressure of the oxygen/argon gas was reduced to 0.1 torr to keep the power flow channel rundown time similar to the hydrogen fill discharges. These data show the complete elimination of the signature of the hot plasma phase.

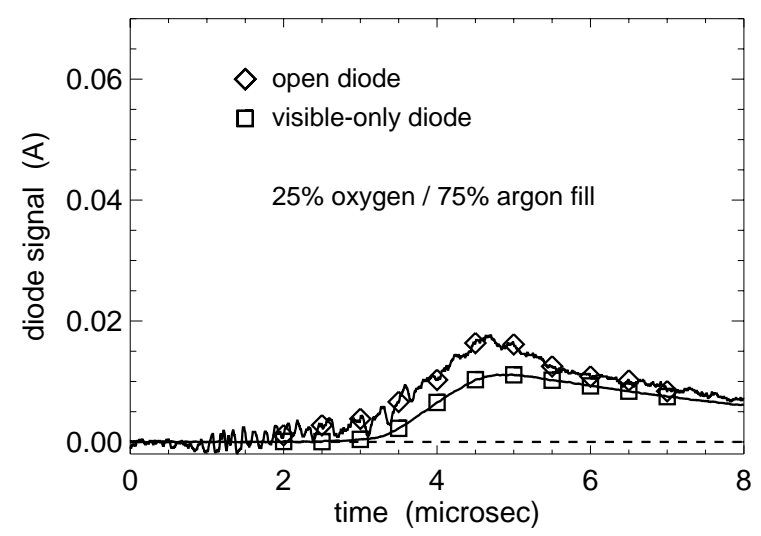

Figure 8. Silicon photodiode data for an open diode and a visible-only diode for a discharge known to be $100 \%$ impure, i.e., a $25 \%$ oxygen / $75 \%$ argon gas fill.

\section{CONCLUSIONS}

The summary data presented here indicates the diffuse z-pinch discharges produced at the Colt facility meet the requirements for magnetic field, plasma density and plasma temperature for a MTF target plasma for a brief period of time, of order $1 \mu \mathrm{s}$. However, the plasma cooling time would appear to be too short for a successful implosion for nominal implosion speeds of $\leq 1 \mathrm{~cm} / \mu \mathrm{s}$.

Originally, a goal of these experiments was to produce discharges with a cryogenic deuterium fiber. This was not possible due to technical problems getting the cryogenic 
deuterium fiber into the inter-electrode gap region. However, the data presented here comparing hydrogen and methane fill and polyethylene fiber discharges give some indication that cryogenic deuterium fiber discharges would not have performed significantly different than the conditions presented here. In particular, there is not a strong indication that the hot plasma lifetime would have been significantly longer.

Since there is not a measure of the impurity content in the hydrogen plasma, it is not clear if the short lifetime can be attributed to impurity radiation or not. However, an estimate of the fraction of plasma energy lost by radiation can be made. The energy content of a $5 \mathrm{~T}, 10^{18} \mathrm{~cm}^{-3}, 150$ $\mathrm{eV}$ plasma filling the containment region is $\approx 2 \mathrm{~kJ}$. The estimated radiation energy for hydrogen fill discharges is $0.6 \mathrm{~kJ}$ [3], which also assumes uniform plasma conditions. This would imply only $30 \%$ of the plasma energy is lost to radiation. Of course this is subject to profile effects and other uncertainties.

Initial analytic estimates of the energy loss due to the axial electron current flow into the end wall gives a cooling time roughly ten times longer than observed.

Analytic 1-D planar estimates of the cross-field ion thermal conduction energy loss to the outer wall indicate that the outer $15 \%$ of the radial extent of the plasma could be cooled in $1 \mu$ s, which accounts for $30 \%$ of the plasma volume. Considering parallel electron thermal conduction to a cold spot along the outer magnetic field lines due to an asymmetry in the magnetic field could also cool a similar $30 \%$ of the plasma volume in $1 \mu$ s.

Analytic 1-D estimates of the energy loss due to unmagnetized ions near the geometric axis (where the magnetic field goes to zero) cooling to the top and bottom end walls indicates that the inner $45 \%$ of the radial extent of the plasma could be cooled in $1 \mu \mathrm{s}$, which accounts for $20 \%$ of the plasma volume.

Thus, it may be possible to explain $50 \%$ of the energy loss by classical thermal conduction. If radiation accounts for the remaining $50 \%$, then the observed hot plasma lifetime may be understandable. Further work is required to fully clarify this scenario.

From an MTF perspective, the data presented here would suggest that other alternate target configurations should be explored, such as the spheromak and/or fieldreversed-configuration (FRC).

\section{REFERENCES}

[1] I. R. Lindemuth and R. C. Kirkpatrick, "Parameter Space For Magnetized Fuel Targets In Inertial Confinement Fusion", Nuclear Fusion 23, 263 (1983).

[2] R. C. Kirkpatrick, I. R. Lindemuth, and M. S. Ward, Fusion Technology 27, 201 (1995).

[3] J. M. Taccetti, F. J. Wysocki, G. Idzorek, H. Oona, R. C. Kirkpatrick, I. R. Lindemuth, and P. T. Sheehey,
"Measurement Of MTF Target Plasma Temperature Using Filtered Photodiodes", in this proceedings.

[4] F. J. Wysocki, R. E. Chrien, G. Idzorek, H. Oona, D. O. Whiteson, R. C. Kirkpatrick, I. R. Lindemuth, and P. T. Sheehey, "Progress With Developing A Target For Magnetized Target Fusion", Digest of Technical Papers for the $11^{\text {th }}$ IEEE International Pulsed Power Conference, Baltimore, Maryland, June 29 to July 2, 1997, G. Cooperstein and I. Vitkovitsky editors, p. 1393.

[5] F. J. Wysocki, J. M. Taccetti, G. Idzorek, H. Oona, R. C. Kirkpatrick, I. R. Lindemuth, and P. T. Sheehey, "Characterization Of A Target Plasma For MTF", Proceedings of the VIII-th International Conference on Megagauss Magnetic Field Generation and Related Topics, Tallahassee, Florida, October 18 to 23, 1998. 